\section{La cultura organizacional y la producción de conocimientos científicos, en maestristas y doctorandos, en la Facultad de Ciencias Administrativas (FCA) de la Universidad Nacional Mayor de San Marcos (UNMSM)-Año 2020, Lima-Perú}

Organizational culture and the production of scientific knowledge, in masters and doctoral students, at the Faculty of Administrative Sciences (FCA) of the Universidad Nacional Mayor de San Marcos (UNMSM) - Year 2020, Lima-Peru

\begin{abstract}
RESUMEN
En la investigación se identifica la influencia de la cultura organizacional en el desempeño de la producción de conocimientos científicos, en maestrías y doctorados, mediante un esquema teórico de espacios generacionales. Considerando el método estadístico del censo, se encuestó a un total de 391 maestristas y doctorandos- Año 2020. Según los resultados, podemos afirmar que la influencia de la Cultura organizacional en la producción de conocimientos científicos, presenta un logro de Nivel Bueno en la formación académica de maestristas y doctorandos, para la producción de tesis. Palabras clave: Cultura organizacional; influencia; producción; conocimientos científicos.
\end{abstract}

\begin{abstract}
This research identifies the influence of the organizational culture in the performance of the production of scientific knowledge, in masters and doctorates, through a scheme of generational spaces. Considering the statistical method of the census, a total of 391 masters and doctoral students were surveyed-Year 2020. According to the results, we can affirm that the influence of Organizational Culture in the production of scientific knowledge presents an achievement of Good Level in the academic training of masters and doctoral students, for the production of theses.
\end{abstract}

Keywords: Organizational culture; influence; production; scientific knowledge.
Sara María Manchego

Odar

smanchegoo@unmsm.edu.pe

Universidad Nacional Mayor de San Marcos, Facultad de Ciencias Administrativas. Lima, Perú

(C) Los autores. Este artículo es publicado por la revista Gestión en el Tercer Milenio de la Facultad de Ciencias Administrativas Universidad Nacional Mayor de San Marcos. Este es un artículo de acceso abierto, distribuido bajo los términos de la licencia Creative Commons Atribución 4.0 Internacional (CC BY 4.0) [https://creativecommons.org/licenses/by/4.0/deed.es] que permite el uso, distribución y reproducción en cualquier medio, siempre que la obra original sea debidamente citada de su fuente original. 


\section{INTRODUCCIÓN}

Cuando una organización se vuelve una permanencia institucional, como el caso de la UNMSM, estos modos aceptables de comportamiento se vuelven evidentes para sus miembros (maestristas y doctorandos), y con ello la Escuela de Posgrado conforma una cultura de organización, por la manera en cómo se creó, para qué se creó, de la manera en que se mantuvo y se mantiene actualmente, y cómo de estos hechos aprende y mejora, además de su habilidad de explicar y predecir el comportamiento de la organización para con los ingresantes y egresados de la Escuela de Posgrado, y ciertamente, por el valor que se crean y vienen creándose en ellos mismos.

Para Amorós (2016), la cultura organizacional, no puede ser rígida sino flexibles, prácticas y apoyadores, por la innovación o, también se estima, conservadoras en su posterior cultura.

Según Gómez-Díaz y Rodríguez (2019), "la cultura organizacional es definida por un conjunto de características que distinguen una organización de otra, que nos informa de cómo las personas en la organización deben comportarse de acuerdo a un sistema de normas y valores" (pp. 1-2).

En principio, en la Escuela de Posgrado, los maestristas y doctorandos son personas más productivas, $y$ al estar insertos en un ambiente globalizado, caracterizado por el predominio del conocimiento como recursos de gestión, la principal ventaja competitiva de las organizaciones como la UNMSM, reside en lograr mejorar a través de la Escuela de Posgrado, el potencial humano y la producción de conocimientos, como indicador fundamental para alcanzar niveles de excelencia en el desarrollo de investigación.

Pese a la importancia de la productividad, eficiencia y eficacia, competitividad, capacidad de adaptación y los factores de satisfacción/insatisfacción de los maestristas y doctorandos, en la escuela de administración de posgrado de la UNMSM, aún no se cuenta con estudios que revelen el grado de dicha satisfacción/insatisfacción en el Desempeño de Producción de Conocimientos (tesis) de los maestristas y doctorandos, y cómo esta satisfacción/insatisfacción puede hallarse influida en un mayor porcentaje por la cultura organizacional propia de la escuela de Posgrado.

Por ello en la investigación se considera importante evaluar que, en esta permanencia institucional, los integrantes de la organización producen, y han producido cambios relacionado a la producción de conocimientos (tesis), y en la metodología de la investigación cuantitativa, cualitativa y mixta. Por ello, la figura del actual docente investigador, planteado por la nueva Ley Universitaria, representa una tentativa gubernamental para que los directores de Escuela de Posgrado, docentes y sus miembros maestristas y doctorandos, mejoren generacionalmente, su funcionamiento en toda la organización.

Los conceptos de Cultura Organizacional y Desempeño de Producción de Conocimientos de los maestristas y doctorandos, son bastante complejos, pero de amplio contenido significativo en las organizaciones como la UNMSM, porque es una realidad constituida por muchos factores. Robbins (2013) define a la cultura organizacional, como un sistema de significado compartido entre sus miembros y que distingue a una organización educativa de las otras, se puede argumentar que la cultura organizacional en la UNMSM, como variable causal, se ocupa en la producción de conocimientos científicos, a través de las siguientes áreas:

- La Facultad de Administración, en lo prescriptivo o normativo, en el quehacer de los encargados de la Facultad, se dirige normalmente a lograr identificar la cultura organizacional contemporánea, que les permita mejorar los diferentes aspectos de eficiencia, competitividad, rentabilidad, capacidad de adaptación; $\mathrm{y}$ ha evaluado las percepciones de los maestristas y doctorandos, identificada previamente por el término descriptivo y/o inferencial de las investigaciones, asesorando según tiempos adecuados en la aprobación de temas, y planes de tesis, que permiten lograr el desempeño de producción de conocimientos de los maestristas y doctorandos.

- El concepto de Cultura Organizacional, comprende un sistema de significado compartido entre sus miembros 
La cultura organizacional y la producción de conocimientos científicos, en maestristas y doctorandos, en la Facultad...

que distingue a una organización como la escuela de Posgrado de Administración de la UNMSM, que parte de un conjunto de valores y supuestos básicos expresados en elementos simbólicos que expresan la capacidad de ordenar, el de atribuir significados, y el lograr construir la identidad organizacional mediante actuaciones de las personas y la instrumentalización de sus relaciones de dominio.

En esta perspectiva, la presente investigación aporta un enfoque teórico y metodológico basado en las competencias centrales de la problemática de la Cultura organizacional y el Desempeño de los docentes, para la problemática planteada de lograr la Producción de Conocimientos Científicos.

El momento actual que viven nuestras sociedades, actualmente con la implementación de la Nueva Ley Universitaria en un ambiente de pandemia denominada COVID-19, se está generando en el Perú, un nuevo espacio-tiempo generacional (Sánchez, 2020), para las actuales comunidades educativas cuyo quehacer es la producción de conocimientos (tesis), y como producto de ello, debe lograrse en un mediano plazo una mejora y aplicación de las rutas de investigación científica, que obliga a los actuales investigadores, el evaluar sus esquemas filosóficos, en la cual la ética y los valores metodológicos, deben ser los garantes de una significativa influencia en el desempeño de la producción de conocimientos científicos.

Para Melían (2017), "mejorar la actual filosofía de la cultura organizacional, para que todos y cada uno de sus integrantes presenten una actitud de cambio para mejorar trámites y planes de estudio" (p. 24). Estas soluciones permitirán elevar los niveles de prestigio y de imagen de la escuela de Posgrado, así como evaluar el desempeño de los docentes de la escuela mediante la filosofía de la cultura organizacional, con estrategias educacionales de metodología y estadística, enfocadas en tiempo real, dirigida a la Sostenibilidad de la educación universitaria de la UNMSM, en la Producción de Conocimientos.

Por otro lado, Tamayo y Romero (2019), citando a Robbins y Coulter (2013), mencionan que la "mayoría de las empresas (universidades) buscan establecer un ambiente que les ayude a mantener sus niveles de productividad para sobrevivir como empresas (universidades), adecuarse a los cambios y resolver problemas, desafíos y retos, y para que esto tenga éxitos" (p. 28). Cada uno dentro de la organización en mayor o menor grado marca la diferencia en su comportamiento, en su entendimiento y estas características generalmente no están en el consciente de los integrantes de la organización; sin embargo, cuando se habla de cultura organizacional se trata de conocer cómo sus integrantes ven su organización, cómo esta los estimula y cómo aprecian su futuro, con qué expectativas o dudas en los ambientes laborales en que se desempeñan.

\section{MÉTODOS}

En Metodología, se requiere, en primer lugar, el conocer, describir, evaluar, explicar y discutir los fundamentos filosóficos, epistemológicos, ontológicos, de valor contenido en cada paradigma o ruta de investigación. En segundo lugar, si el investigador tiene alguna preferencia por un paradigma aplicará la metodología (enfoque) según el método elegido, iniciándose con la descripción de la realidad problemática, la cual deberá estar fundamentada mediante el enfoque a utilizar en la investigación (el cuantitativo, el cualitativo, el método mixto).

Hernández y Mendoza (2018), definen “investigación como un conjunto de procesos sistemáticos de la administración, críticos y empíricos para generar conocimientos utilizando diseños y/o enfoques cuantitativos, cualitativos y mixtos, para el reporte de sus resultados" (p.14). En el diseño se debe incluir un diagnóstico de la escuela de posgrado para determinar el estado real de la gestión en la producción de conocimientos, que permita comprobar, los niveles alcanzados en productividad y competitividad con otras escuelas, con el cual es posible consolidar un modelo básico de gestión y producción del conocimiento.

Con relación a la producción del conocimiento este puede ser el elemento generador de la ventaja competitiva de la escuela de posgrado, al gestionar conocimiento en investigación científica, por los siguientes argumentos: Mejor comprensión de los resultados de la estadística, 
mejorando cuatro lecturas, dato, información, explicación y conocimiento. Luego, el aplicar un diseño estadístico (Sánchez, 2020) denominado estadística circular (correlación, prueba $t$, análisis de varianza $\mathrm{F}$, y la chi cuadrado), para evaluar y explicar resultados con originalidad y creatividad para interpretar la realidad problemática del momento, plantear recomendaciones y/o propuestas cuantitativas, cualitativas o mixtas, para la toma de decisiones.

La investigación está relacionada con el enfoque cuantitativo porque aplica la estadística para la prueba de hipótesis donde se apreciará su falsedad o veracidad, asimismo "se utiliza la recolección y el análisis de datos para dar respuesta al problema de la investigación" (Sánchez, Guillen y Bagazo, 2020, p. 31).

El procedimiento en el contraste de la hipótesis se apoya en la utilización del SPSS versión 27 , con el ingreso de los resultados de las encuestas y con las rutas correspondientes relacionados a la regresión logística ordinal, se halla el dato del valor sig. bilateral, el cual si arroja un valor menor al 0.005 se acepta la hipótesis alterna y si el valor es mayor o igual a 0.005 se acepta el valor nulo. Con el ingreso de los datos, se procede a "la distribución de frecuencias que es un conjunto de puntuaciones de una variable ordenada en sus respectivas categorías" (Hernández \& Mendoza, 2018, p. 328).

\section{RESULTADOS}

Teniendo en cuenta la revisión de la literatura existente y para evaluar los resultados de la investigación, en el contraste de hipótesis, se utilizó el método de correlación de Pearson, que fue de 0.672 . La distribución beta, determinada para la variable producción de conocimientos científicos en la Escuela de Postgrado de la UNMSM, para la Condición de estudio fue de: Beta $=0.453$, seguido de la Identidad Organizacional: Beta $=0.267$, y la sostenibilidad organizacional: Beta $=0.235$.

En la Cultura organizacional, la eficacia alcanzó un Beta $=0.446$, seguido de la Productividad: Beta $=0.419 ; \mathrm{y}$ de la Satisfacción: Beta $=$ 0.200 . En la Producción de conocimientos para la compatibilidad emocional: Beta $=0.324$, seguida de la Sostenibilidad Organizacional: Beta $=0.308$.

\section{DISCUSIÓN}

Los resultados de la investigación, Influencia de la Cultura Organizacional en la Producción de Conocimientos científicos, se analizan según los niveles causa-efecto alcanzados, hallándose las siguientes relaciones.

- La relación causa- efecto de la Identidad Organizacional y Condiciones de Estudio con la Eficacia, presenta un Nivel de Alto.

- La relación causa- efecto de la Sostenibilidad Organizacional con la Satisfacción, presenta un Nivel de Bueno.

- Las relaciones Compatibilidad Organizacional con la Capacidad de Adaptación, presentan un Nivel de Regular.

De estos resultados, podemos afirmar que la influencia de la Cultura organizacional en la Producción de conocimientos científicos, presenta un logro de Nivel Bueno en la formación académica de maestristas y doctorandos, para la producción de tesis.

Los resultados evidencian la influencia significativa de la Cultura organizacional en la Producción de conocimientos científicos $(r=0.672)$, siendo nuestros resultados utilizando la correlación de Pearson obtenidas, más altas que las observadas por Quispe y Tinoco (2014) entre cultura organizacional y satisfacción laboral.

\section{CONCLUSIONES}

1. De acuerdo al criterio teórico, ha quedado demostrado que la Cultura Organizacional, según la Identidad Organizacional, influye en el desempeño de la producción de conocimientos científicos, en maestristas y doctorandos de la Facultad de Administración de la Universidad Nacional Mayor de San Marcos - Año 2020, Lima- Perú.

2. De acuerdo al criterio teórico, ha quedado demostrado que la Cultura Organizacional, según Incentivos y Recompensas, no influye en el desempeño de la producción de conocimientos científicos, en maestristas y doctorandos. 
La cultura organizacional y la producción de conocimientos científicos, en maestristas y doctorandos, en la Facultad...

3. En relación a las Condiciones de Estudio, la cultura organizacional influye en el desempeño de la producción de conocimientos científicos, en maestristas y doctorandos.

4. La Cultura Organizacional, considerando la Sostenibilidad Organizacional, se relaciona con el desempeño de la producción de conocimientos científicos, en maestristas y doctorandos.

5. La Cultura Organizacional no influye en la Compatibilidad Emocional, no influye en el desempeño de la producción de conocimientos científicos, en maestristas y doctorandos, de la Facultad de Administración de la Universidad Nacional Mayor de San Marcos - Año 2020, Lima- Perú.

6. Como conclusión final, la Cultura Organizacional influye en el desempeño de la producción de conocimientos científicos, en maestristas y doctorandos de la Facultad de Administración de la Universidad Nacional Mayor de San Marcos - Año 2020, Lima- Perú.

\section{REFERENCIAS BIBLIOGRÁFICAS}

Amorós, E. (2016). Comportamiento Organizacional. En busca del Desarrollo de Ventajas Competitivas. Reimpresión autorizada a la Universidad Católica Santo Toribio de Mogrovejo. Lambayeque. Perú.
Bernal, C. (2016). Metodología de la investigación. Bogotá: Pearson.

Gómez Díaz, C. y Rodríguez Ortiz, J. (2019). Teorías de la Cultura Organizacional. Actualización, 2019.

Hernández, R. y Mendoza, C. (2018). Metodología de la investigación: las rutas cuantitativa, cualitativa y mixta. Primera edición. McGraw Hill Interamericana Editores, S.A. México.

Melián, V. (2017). La cultura organizacional y su impacto en el rendimiento de los equipos de trabajo: el papel mediador del clima y la reflexividad. Facultad de Psicología. Valencia. España.

Robbins, S. (2013). Comportamiento internacional. Décimo quinta edición. Pearson Education, Inc. México.

Sánchez, M., Guillen, O. y Begazo, L. (2020). Pasos para elaborar una tesis de tipo correlacional. Bajo enfoque cuantitativo, variable categórica y la estadística no paramétrica. Lima: Oscar Guillen Valle.

Sanchez S., S. (2020) Percepción de la neuroestructura en investigación científica: el espacio tiempo. Imprenta UNFV (en impresión).

Tamayo, Ñ. y Romero, C. (2019). El clima laboral y el desempeño de los colaboradores de la Superintendencia de Mercado de valores, Miraflores. Universidad Nacional de Educación Enrique Guzmán y Valle. Lima, Perú. 
